\title{
Clinical Study \\ Benefit of Contact Force Sensing Catheter Technology for Successful Left Atrial Anterior Line Formation: A Prospective Randomized Trial
}

\author{
Stephanie Fichtner $\left(\mathbb{D},{ }^{1}\right.$ Reza Wakili, ${ }^{1,2,3}$ Konstantinos Rizas, ${ }^{1,2}$ \\ Johannes Siebermair, ${ }^{1,2,3}$ Moritz F. Sinner, ${ }^{1,2}$ Thomas Wiktor, ${ }^{1}$ \\ Korbinian Lackermair ${ }^{D},{ }^{1}$ Franziska Schuessler, ${ }^{4}$ Lucia Olesch, ${ }^{1}$ \\ Susanne Rainer, ${ }^{1}$ Stefan Kääb, ${ }^{1,2}$ Adrian Curta, \\ Harald Kramer, ${ }^{5}$ and Heidi L. Estner ${ }^{1}$ \\ ${ }^{1}$ Department of Medicine I, University Hospital Munich, Ludwig-Maximilians University, Munich, Germany \\ ${ }^{2}$ German Cardiovascular Research Centre (DZHK), Partner Site: Munich Heart Alliance, Munich, Germany \\ ${ }^{3}$ Department of Cardiology and Vascular Medicine, West-German Heart and Vascular Center Essen, \\ Essen University Hospital, University Duisburg-Essen, Essen, Germany \\ ${ }^{4}$ Charité Campus Virchow-Klinikum, Berlin, Germany \\ ${ }^{5}$ Institute for Clinical Radiology, University Hospital Munich, Ludwig-Maximilians University, Munich, Germany \\ Correspondence should be addressed to Stephanie Fichtner; stephanie.fichtner@med.uni-muenchen.de
}

Received 20 June 2018; Revised 9 August 2018; Accepted 19 August 2018; Published 18 September 2018

Academic Editor: Natale Daniele Brunetti

Copyright (C) 2018 Stephanie Fichtner et al. This is an open access article distributed under the Creative Commons Attribution License, which permits unrestricted use, distribution, and reproduction in any medium, provided the original work is properly cited.

Introduction. The value of contact force information for ablation of LA anterior line is unknown. In a prospective randomized clinical trial, we investigated if information on contact force during left atrial (LA) anterior line ablation reduces total radiofrequency time and results in higher rates of bidirectional line block in patients undergoing pulmonary vein isolation (PVI) plus substrate modification. Methods. We included patients with indication for pulmonary vein isolation (PVI) and additional substrate modification. For LA anterior line ablation, patients were randomized to contact force information visible $(\mathrm{n}=35)$ or blinded $(n=37)$. Patients received contrast enhanced cardiac magnetic resonance imaging (cMRI) before and 3-6 months after ablation to visualize the LA anterior line. Primary endpoint was radiofrequency time to achieve bidirectional line block. Secondary endpoints were completeness of the LA anterior line on cMRI, distribution of contact force, procedural data, adverse events, and 12 months success rate. Results. In 72 patients (64 \pm 9 years, $68 \%$ male), bidirectional LA anterior line block was achieved in 70 (97\%) patients. Radiofrequency time to bidirectional block did not differ significantly across groups (contact force information visible $23 \pm 18 \mathrm{~min}$ versus contact force information blinded $21 \pm 15 \mathrm{~min}, \mathrm{p}=0.50)$. The LA anterior line was discernable on cMRI in 40 patients ( $82 \%)$ without significant differences across randomization groups $(\mathrm{p}=0.46)$. No difference in applied contact force was found depending on cMRI line visibility. Twelve-month success and adverse event rates were comparable across groups. Conclusion. Information on contact force does not significantly improve the ablation of LA anterior lines. Clinical Trial Registration. The trial was registered at http://www.clinicaltrials.gov by identifier: NCT02217657.

\section{Introduction}

Catheter ablation of atrial fibrillation (AF) has evolved as a guideline recommended therapeutic option for patients with this condition. Whereas wide area circumferential pulmonary vein isolation (PVI) comprises the standard approach, selected patients require additional left atrial (LA) linear lesions [1]. By applying LA linear lesions, the goal is to achieve bidirectional conduction block, as incomplete block predisposes to the development of atypical atrial flutter. 
However, depending on the LA anatomy, bidirectional block of the anterior line can only be achieved in $60 \%$ to $86 \%[2,3]$.

Preclinical research showed that appropriate contact force between the ablation catheter tip and the target tissue is a key contributor to effective lesion formation $[4,5]$. Insufficient contact may result in an ineffective lesion, leading to arrhythmia recurrence, whereas excessive contact force may result in tissue damage and possibly life threatening complications [5]. In multicenter clinical trials, PVI using contact force sensing catheter technology resulted in a reduced rate of immediate, intraprocedural pulmonary vein reconnection [6], and reduced rates of AF recurrence during follow-up [7]. However, the benefit of using contact force sensing catheter technology is unclear for the formation of LA linear lesions.

Thus, in our study, we included patients with persistent $\mathrm{AF}$ or AF recurrence after a first PVI and randomized them to receive a LA anterior line ablation using either visible or blinded contact force sensing information. Beyond measures of procedural success, we systematically obtained cardiac magnetic resonance imaging (cMRI) to visually assess the ablation success.

\section{Methods}

2.1. Study Cohort. Patients scheduled for catheter ablation of drug refractory persistent AF or for catheter reablation of AF recurrence after PVI for paroxysmal AF were eligible for study participation because of needed additional substrate modification. We excluded patients for the following reasons: left atrial thrombus, symptomatic mitral valve stenosis or moderate to severe mitral valve insufficiency $(\geq$ grade 2 of 4), severely impaired left ventricular function (left ventricular ejection fraction $\leq 35 \%$ ), hyperthyroidism, current pregnancy, and age $<18$ years or $>80$ years. All patients provided written informed consent. The ethics committee at the Ludwig-Maximilians University, Munich, Germany, approved the study, which was also registered at www.clinicaltrials.gov (NCT02217657).

After written informed consent, through an investigator not involved in the ablation procedure, all enrolled patients received a wide area circumferential PVI and were intraprocedurally randomized in a 1:1 fashion to receive a left atrial anterior line either with or without real-time display of catheter tip-to-tissue contact force sensing information to the investigator. Randomization was undertaken using sealed envelopes. Figure 1 summarizes the study procedures.

Our primary endpoint was the radiofrequency time to achieve bidirectional block of LA anterior line. Secondary endpoints were procedural duration, fluoroscopy time, and radiation dose and differences in contact force measures between both groups. We further visually assessed LA anterior line lesion formation by cMRI. Over the follow-up duration of 12 months, we also compared the occurrence of adverse events and the postprocedural atrial arrhythmia recurrence rate.

2.2. Ablation Procedure. Patients were kept on continuous oral anticoagulation, aiming for an international normalized ratio (INR) of 2.0-2.7. For patients using Dabigatran, Apixaban, or Rivaroxaban, the last dose was paused. Ablation procedures were performed under conscious sedation with midazolam and remifentanil. For electroanatomical mapping and three-dimensional navigation, we used the Carto 3 mapping system (Biosense Webster Inc., Diamond Bar, CA, USA). A 10-electrode deflectable catheter was placed in the coronary sinus (Inquiry, St. Jude Medical, LLC, St. Paul, MN, USA), and the aortic root was marked using a pigtail catheter introduced via the radial artery. The LA was accessed by single or double transseptal puncture via a steerable sheath (Agilis, St. Jude Medical, LLC, St. Paul, MN, USA). A fluoroscopic angiogram of all pulmonary veins was performed prior to the ablation. During the LA procedure time, intensified anticoagulation was maintained with unfractionated heparin aiming for an activated clotting time of $\geq 300$ seconds.

For PVI, a steerable 20 electrode circular mapping catheter (Lasso, Biosense Webster Inc., Diamond Bar, CA, USA) was placed inside the pulmonary veins via the steerable sheath. Radiofrequency (RF) ablation was then performed using an open irrigated tip ablation catheter with contact force sensing capabilities (Smart Touch, Biosense Webster Inc., Diamond Bar, CA, USA), mostly without usage of a sheath. Catheter settings were as follows: 30 watts, power controlled with a temperature maximum of $43^{\circ}$ Celsius, and an irrigation rate of $30 \mathrm{ml} / \mathrm{min}$. We aimed for an ipsilateral wide antral circumferential isolation of all pulmonary veins in all patients regardless if PVI was performed previously. RF lesions were done using the dragging method between ablation points. Main target for individual lesion was electrogram diminution with a contact force above $10 \mathrm{~g}$. Pulmonary vein isolation was confirmed by documentation of entrance and exit block interpreting the bipolar electrograms of the circular mapping catheter.

Following successful PVI, all patients received a LA anterior line, depending on randomization using visible or blinded contact force sensing information, since 10/2013 Visitag was available and not displayed in the blinded group. The LA anterior line was formed using the same RF ablation catheter as for PVI, connecting the anterior mitral annulus with the left superior pulmonary. LA anterior line formation was considered successful by documentation of bidirectional block using differential pacing criteria and by documentation of double potentials along the line $[3,8]$. In the group with contact force displayed, real-time contact force was visible throughout the procedure, and investigators aimed for $10-30 \mathrm{~g}$ [9]. In the contact force blinded group, investigators relied on standard criteria for ablation guidance. No other line apart from LA anterior line was performed.

After a waiting period of 30 minutes after successful formation of the LA anterior line, PVI isolation was reconfirmed including fractionated application of intravenous adenosine for each PV to rule out dormant conduction [10]. At the LA anterior line, bidirectional conduction block was reconfirmed.

2.3. Cardiac Magnetic Resonance Imaging. cMRI was performed with a 3-Tesla system (Magnetom Verio, Siemens 


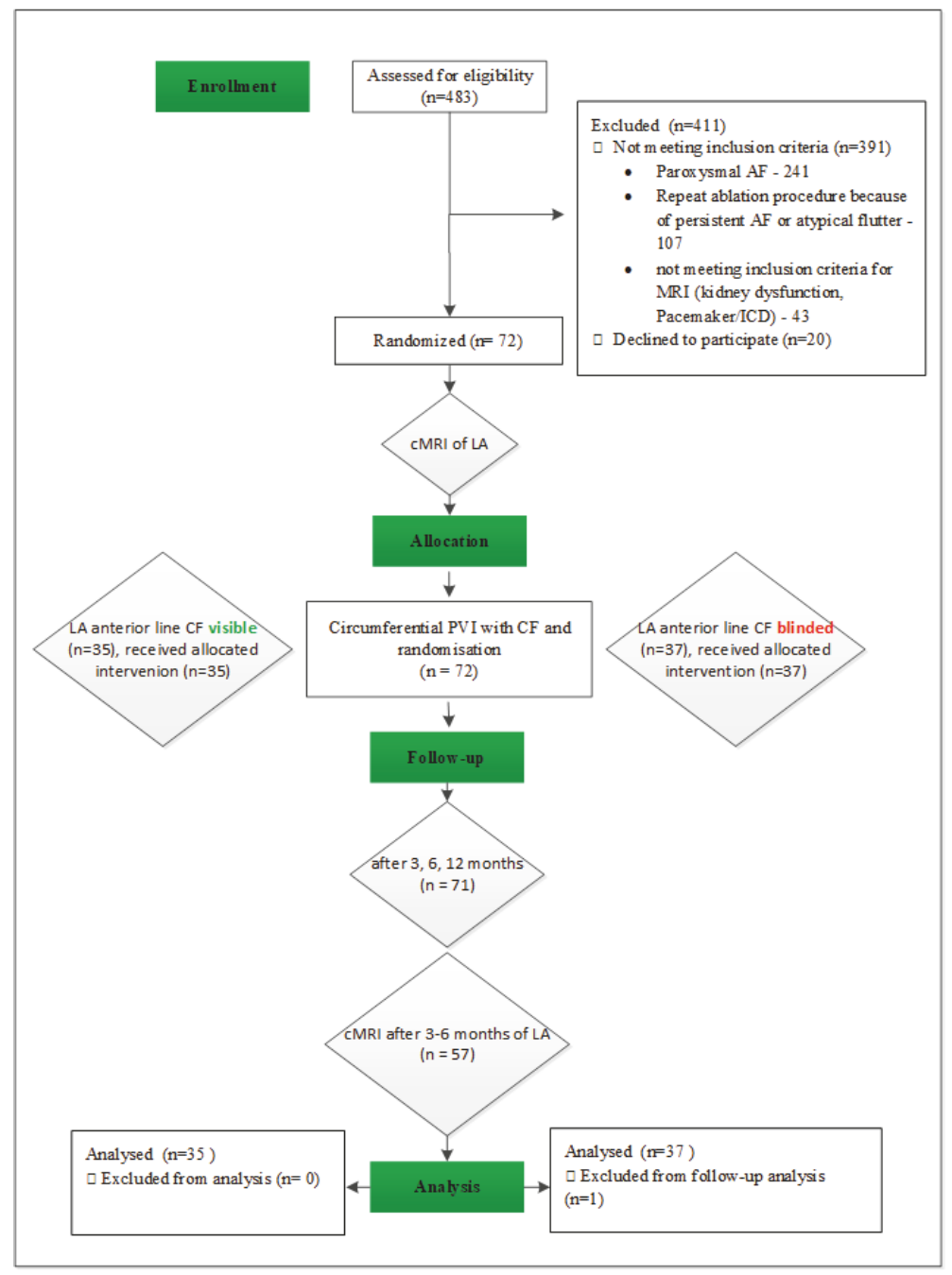

FIgURE 1: Study flowchart. Ext CV: external cardioversion; AF: atrial fibrillation; cMRI: cardiac magnet resonance imaging; LA: left atrium; CF: contact force; PVI: pulmonary vein isolation.

Healthineers, Erlangen, Germany). A first cMRI was performed prior to the ablation procedure to visualize preexisting scar tissue in the ablation region. To improve image quality, patients in AF underwent external cardioversion prior to the scan. A second cMRI was scheduled three to six months after the ablation for visualization of the anterior line.

Methodologically, fast low angle shot-inversion recovery sequences (FLASH-IR) were performed 15 minutes after contrast agent application (Gadobutrol $0.15 \mathrm{mmol} / \mathrm{kgbw}$, Gadovist, BayerSchering, Berlin, Germany) ( $\mathrm{ST}=0,9 \mathrm{~mm}$, $\mathrm{TE}=1.460 \mathrm{~ms}, \mathrm{TR}=462.560 \mathrm{~ms}, \mathrm{FA}=20^{\circ}$ ) for analysis of ablation lesions by late gadolinium enhancement (LGE). Two investigators blinded to randomization status and clinical parameters independently assessed the LA anterior line visually comparing both available cMRI scans using the Siemens Argus Software (Siemens Healthineers, Erlangen, Germany). In case of discrepancies, a final judgment was reached by consent. LA anterior line assessment was standardized by defining three distinct line segments that were assessed individually: (a) anterior mitral valve annulus to inferior left atrial appendage; (b) septal of the left atrial appendage; (c) roof of the left atrial appendage to the left superior pulmonary vein (Figure 3 ). The same segment based analysis was performed for the secondary analysis of locally applied contact force data.

2.4. Follow-Up. Systematic follow-up was scheduled at the arrhythmia clinic 3, 6, and 12 months after the ablation procedure and done through physicians who were not part of the ablation team and were blinded to treatment group. At each visit, arrhythmia-related symptoms and adverse events were surveyed. A 7-day Holter-ECG was obtained at each visit. For AF recurrence analysis, we considered a blanking period of 3 months. After this period, AF recurrence was defined as any documented atrial arrhythmia of $\geq 30$ second's duration. [1] Because the subgroup of patients with repeat ablation due to relapse of paroxysmal AF was too small, only patients with persistent AF and first ablation procedure were analyzed regarding sinus rhythm after 12 months. Oral anticoagulation was discontinued in patients without $\mathrm{AF}$ 
TABLE 1: Baseline characteristics.

\begin{tabular}{lccc}
\hline & Contact force information visible & Contact force information blinded & P value \\
\hline Age (years) & $66 \pm 9$ & $62 \pm 9$ & 0.06 \\
Sex (male) & $24(65 \%)$ & $25(71 \%)$ & 0.62 \\
AF duration (years) & $4.8 \pm 3.1$ & $3.7 \pm 3.7$ & 0.21 \\
Persistent AF & $28(78 \%)$ & $32(91 \%)$ & 0.19 \\
LA diameter (mm) & $43.6 \pm 5$ & $43.8 \pm 5$ & 0.85 \\
EHRA Score (mean) & $2.3 \pm 0.9$ & $2.2 \pm 0.9$ & $2.2 \pm 1.6$ \\
CHADS Vasc Score (mean) & $2.2 \pm 1.0$ & $1.1 \pm 0.8$ \\
HAS Bled Score (mean) & $1.4 \pm 0.7$ & $21(60 \%)$ & 0.87 \\
Arterial hypertension (\%) & $22(66 \%)$ & $7(20 \%)$ & 0.99 \\
Vascular disease (\%) & $6(18 \%)$ & $6(17 \%)$ & 0.8 \\
Heart failure (\%) & $4(12 \%)$ & $6(17 \%)$ \\
Previous stroke (\%) & $2(6 \%)$ & $2(6 \%)$ & 0.47 \\
Diabetes (\%) & $2(6 \%)$ & 0.26 \\
\hline
\end{tabular}

Data are mean \pm standard deviation or frequency (percentage). LA: left atrial; EHRA: European Heart Rhythm Association.

recurrence after 6 months in case of a $\mathrm{CHA}_{2} \mathrm{DS}_{2}$-VASc score $<2$.

2.5. Statistical Analysis. We based our power calculation on the experience of a previous study [3]. We assumed a RF application time of 17 minutes for LA anterior line formation without information on contact force. With contact force information available, we expected a $40 \%$ reduction of RF application time (10 minutes) [11]. Aiming for a two-sided $\alpha$ of $5 \%$ and a power of $80 \%$, we calculated a sample size of 62 patients (31 patients per group). To account for a primary success rate of LA anterior line conduction block of $86 \%$, we planned our study to enroll 72 patients (36 patients per group).

Statistical analysis was performed using SPSS (version 24, IBM SPSS Statistics, IBM Corp, Amonk, NY) and R studio ( $\mathrm{R}$ foundation for statistical computing, Vienna Austria). Continuous variables are presented as mean \pm standard deviation and are compared using the Wilcoxon rank sum or Student's $t$-tests. Categorical data are presented as frequency and percentages and are analyzed using the chi-square or Fisher exact tests. In addition, we fitted linear regression models to account for potential confounding by age, sex, and CHA2DS2-VASc score. P values $<0.05$ were considered statistically significant.

\section{Results}

3.1. Patient Characteristics. Between 2012 and 2015, 72 patients were prospectively randomized and included in the analysis. No significant differences in baseline characteristics were noted between both study groups (Table 1).

3.2. Procedural Results. All patients received PVI and additional ablation of a LA anterior line as planned and according to randomization status. PVI was procedurally successfully in all patients (100\%). In 70 of 72 patients (97\%), a bidirectional block of the LA anterior line was achieved. The primary endpoint being radiofrequency time to achieve bidirectional

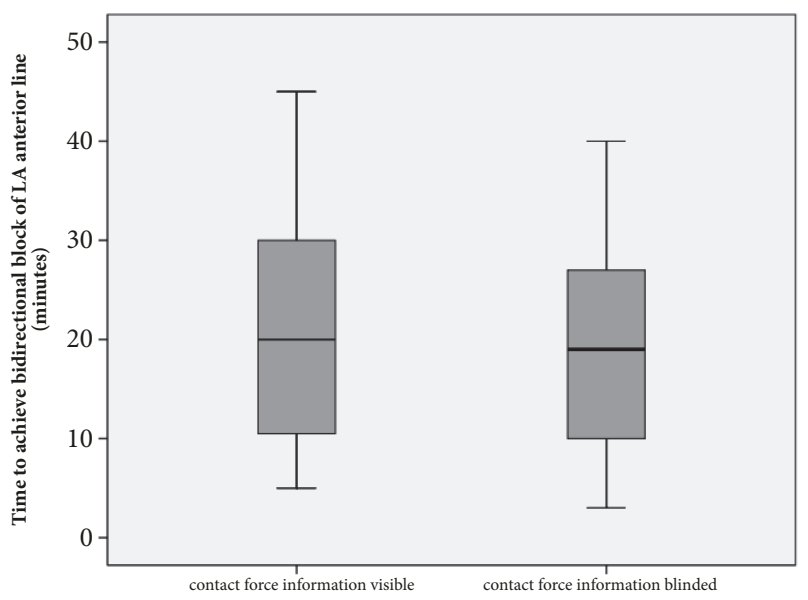

FIgURE 2: The primary endpoint of time to bidirectional block of the LA anterior line did not differ between both groups.

block at the LA anterior line did not differ significantly $(\mathrm{p}=0.50$ ) between both groups with $23 \pm 18 \mathrm{~min}$ (contact force information visible) versus $21 \pm 15 \mathrm{~min}$ (contact force information blinded) (Table 2; Figure 2).

In addition, secondary endpoints did not differ significantly between both groups: overall procedure time (contact force information visible: $192.6 \pm 30 \mathrm{~min}$ versus contact force information blinded: $189 \pm 60 \mathrm{~min}, \mathrm{p}=0.70$ ); overall fluoroscopy time ( $23.3 \pm 10$ min versus $27.9 \pm 13 \mathrm{~min}, \mathrm{p}=0.12)$; overall radiation dose $\left(1930 \pm 2025 \mathrm{cGy} * \mathrm{~cm}^{2}\right.$ versus $1692 \pm 1128$ $\left.\mathrm{cGy} * \mathrm{~cm}^{2}, \mathrm{p}=0.57\right)$. Details are provided in Table 2.

Analysis of contact force information and force time integral at the LA anterior line by prespecified line segments similarly revealed no significant differences between both groups (Figure 3). Across line segments, contact force $>10 \mathrm{~g}$ was achieved in $80 \%$ of applied lesions.

Six patients (16\%) in the visible group and six patients (17\%) in the blinded group showed a reconnected LA anterior line after the waiting period and were successfully reablated 
TABLE 2: Procedural data.

\begin{tabular}{|c|c|c|c|}
\hline & Contact force information visible & Contact force information blinded & P value \\
\hline Procedure time (min) & $192.6 \pm 30$ & $189 \pm 60$ & 0.7 \\
\hline Total fluoroscopic time (min) & $23.3 \pm 10$ & $27.9 \pm 13$ & 0.12 \\
\hline LA anterior line fluoroscopy time ( $\mathrm{min})$ & $1.1 \pm 1.2$ & $0.8 \pm 1.1$ & 0.3 \\
\hline Radiation dose $\left(\mathrm{cGy} * \mathrm{~cm}^{2}\right)$ & $1930 \pm 2025$ & $1692 \pm 1128$ & 0.57 \\
\hline Time to complete LA anterior line (min) & $23 \pm 18$ & $21 \pm 15$ & 0.5 \\
\hline LA anterior line reconnection during waiting period & $6(16 \%)$ & $6(17 \%)$ & 0.9 \\
\hline LA anterior line RF applications (n) & $10 \pm 7$ & $10.4 \pm 11$ & 0.9 \\
\hline
\end{tabular}

Data are mean \pm standard deviation. LA: left atrial; $R F$ : radiofrequency.

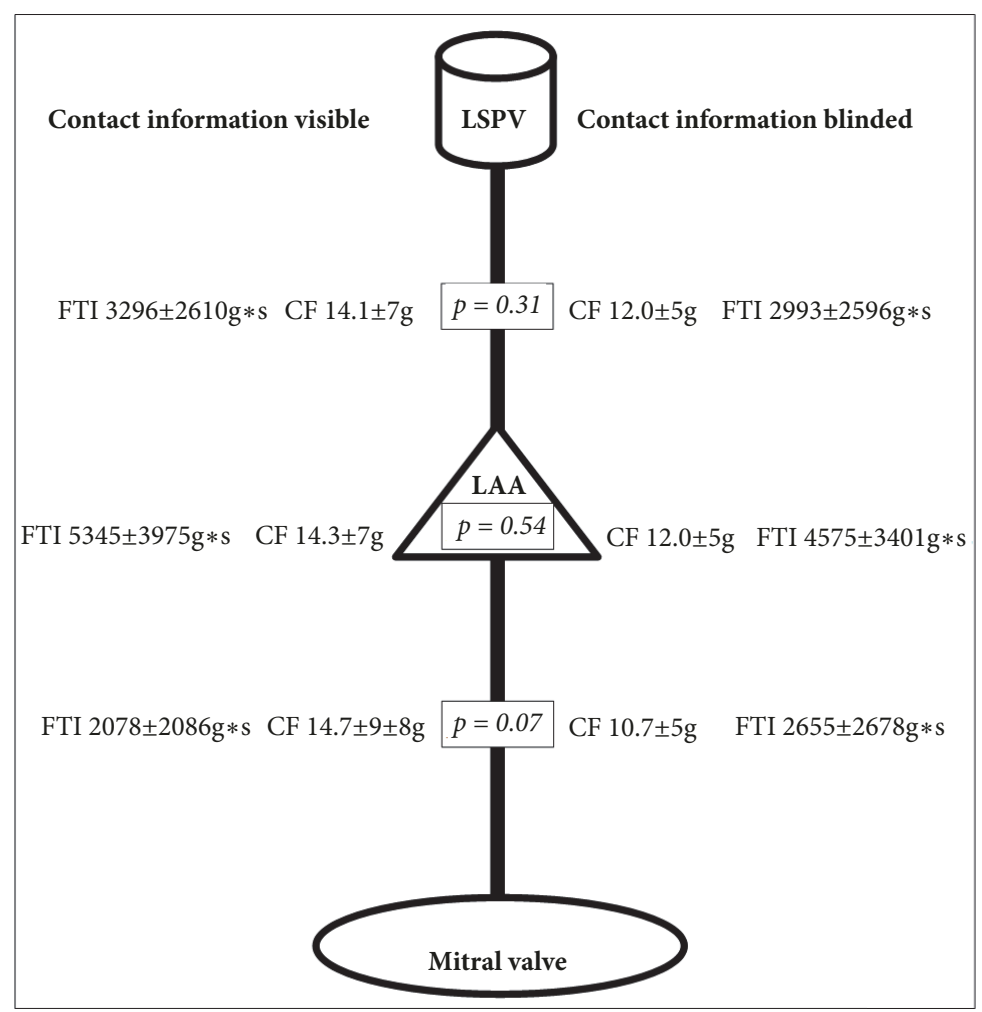

FIGURE 3: Distribution of contact force and force time integral in different segments of the anterior line. LAA: left atrial appendage; CF: contact force; FTI: force time integral; LSPV: left superior pulmonary vein. No significant difference could be detected between groups.

showing no significant difference between both groups. Patients with reconnection compared to patients without showed no significant difference in applied contact force (13.1 $\pm 4 \mathrm{~g}$ versus $13.6 \pm 6 \mathrm{~g}$ respectively, $\mathrm{p}=0.8)$.

The secondary endpoint comparing contact force in three distinct segments of the LA anterior line did not reveal significant differences across randomization groups: LSPV, left superior pulmonary vein; LAA, left atrial appendage; $C F$, contact force (Figure 3).

Multivariable adjusted analyses suggested no confounding by cMRI derived end-diastolic or end-systolic left ventricular volume, LA ejection fraction (cMRI), left ventricular ejection fraction (echocardiography), and ablation in sinusrhythm or AF.

3.3. MRI Results. In 49 patients, both the cMRI acquired at baseline and at follow-up showed sufficient image quality for analysis. The remaining patients were not suitable for analysis due to insufficient image quality $(n=8)$, incident pacemaker implantation after the baseline scan $(n=4)$, and withdrawal of consent for the follow-up cMRI ( $\mathrm{n}=11)$.

In the initial cMRI, LGE at the anterior wall was present in six patients (15\%) without a difference between both groups $(\mathrm{p}=0.19)$. LGE before ablation procedure did not influence time to achieve bidirectional block of anterior line and did not influence outcome after 12-month follow-up. After the ablation, the LA anterior line was visible on cMRI in at least one segment in 40 patients $(82 \%)$, regardless of whether contact force was displayed or not $(\mathrm{p}=0.46)$. Restricting the analysis to those patients with visible LA anterior line on cMRI, we similarly found no significant differences in contact force between randomization groups (contact force information visible: $12.9 \pm 4 \mathrm{~g}$ versus contact force information blinded: 11.6 $\pm 1.8 \mathrm{~g}, \mathrm{p}=0.42$ ) (Figure 3). 
In 26 patients (53\%), which underwent a post-cMRI, a complete LA anterior line was detected by cMRI in all three segments, with no difference between both groups $(p=0.78)$. In addition, no significant differences regarding contact force could be detected (contact force information visible: $14 \pm 7 \mathrm{~g}$ versus contact force information blinded: $12.7 \pm 3 g, p=0.31$ ). Segment-specific analysis revealed that the LA anterior line was visible in $66 \%$ of patients between the anterior mitral annulus and the left atrial appendage, in $61 \%$ of patients septal of the left atrial appendage, and in $75 \%$ of patients between the left atrial appendage and the left superior pulmonary vein. For neither of the segments did we identify significant differences in contact force: contact force information visible: $14.7 \pm 9 \mathrm{~g}, 14.3 \pm 7 \mathrm{~g}$, and $14.1 \pm 7 \mathrm{~g}$, respectively, versus $10.7 \pm 5 \mathrm{~g}, 13.2 \pm 5 \mathrm{~g}$, and $12.0 \pm 5 \mathrm{~g}$, respectively; $\mathrm{p}=0.07, \mathrm{p}=0.54$, and $\mathrm{p}=0.31$, respectively. Multivariable adjustment did not relevantly change these results.

3.4. Follow-Up at 12 Months and Repeat Ablation Procedures. Follow-up at 12 months was available for 71 (99\%) patients, with one patient lost to follow-up. The recurrence rate of any documented atrial arrhythmia in patients with persistent AF off any antiarrhythmic class I or class III medication was $45 \%$ (contact force information visible) versus 52\% (contact force information blinded) after a single procedure $(\mathrm{p}=0.8)$.

Overall, 17 patients underwent repeat ablation, equivalent to $23 \%$ of patients in the contact force information visible group and $29 \%$ of patients in the contact force information blinded group $(\mathrm{p}=0.8)$. The rates of left atrial flutter (contact force information visible: $37 \%$ versus contact force information blinded: $44 \%, \mathrm{p}=0.16$ ) and the percentages of sustained bidirectional block of the LA anterior line during the repeat procedure (contact force information visible: $37 \%$ versus contact force information blinded: $44 \%, \mathrm{p}=0.1$ ) did not differ significantly. In 10 of 17 patients with repeat ablation cMRI was available. Of these, eight patients showed a reconnected LA anterior line during the repeat procedure. In this subgroup, LA anterior line was visible on cMRI in all segments in $3(37 \%)$ patients and was not visible in all segments in $5(63 \%)$ patients showing no statistical difference between groups $(\mathrm{p}=1.0)$.

3.5. Adverse Events. Acute adverse events during the index hospitalization occurred in four patients (5\%). One patient developed cardiac tamponade requiring pericardiocentesis in the contact force information blinded group and could be discharged without any further problems. In this patient, no excessive contact force was recorded. In two patients (one per group), groin vascular access site pseudoaneurysms were treated without necessity for surgical intervention. One patient in the contact force information blinded group developed a groin AV-fistula, which resolved without surgical intervention. No significant difference in adverse event rates was noted between both groups $(p=0.61)$.

\section{Discussion}

In this prospective, randomized clinical trial of patients undergoing PVI for AF, display of contact force information during the application of an additional LA anterior line did not reduce the radiofrequency time to achieve bidirectional block of the line. In addition, contact force, procedural measures, visibility of the LA anterior line on cMRI, and the AF ablation outcome after 12 months of follow-up were similar between both groups.

4.1. Contact Force during Linear Ablation. In this study, 72 patients were randomized to application of a LA anterior line either with or without the display of contact force information. In all patients, the same ablation catheters and mapping systems were used. The primary endpoint of time to bidirectional block of the LA anterior line, as well as various secondary endpoints, did not differ significantly between groups. Until now, no study addressing the efficacy and effectiveness of contact force information on LA linear ablations was available. Prior studies using conventional ablation catheters achieved bidirectional block of LA anterior lines in only $60 \%$ to $86 \%[2,3]$. We thus hypothesized that information on contact force could improve the time to achieve bidirectional block of the LA anterior, but failed to identify significant differences. However, our rate of achieved bidirectional block (97\%) was much higher than previously reported using conventional ablation catheters. Possible explanations may include longer investigator experience in LA line ablation compared to early reports with limited experience, as well as improvements in ablation catheter technology, even without information on contact force.

In multiple studies, use of contact force for PVI has shown a reduction of acute pulmonary vein reconnection [12-14]. However, in most studies either no randomized protocol was used, or the contact force sensing catheter was compared to a different catheter without contact force sensing capability.

Similar to our study, Ullah et al. randomized 117 patients undergoing PVI with or without information on contact force using the same ablation catheter (Smart-Touch, Biosense Webster) [6]. The primary endpoint was time to achieve complete PVI. In their study, time to achieve PVI, procedure time, and fluoroscopic data did not differ significantly between both groups; in addition, mean contact force was identical in both groups with $13.4 \mathrm{~g}$. However, time in contact force target range $(5-40 \mathrm{~g})$ was significantly higher $(80 \%$ versus $68 \%, \mathrm{p}<0.001)$ and the rate of acute PV reconnection was significantly lower $(22 \%$ versus $32 \%, \mathrm{p}=0.03)$ in those with versus those without display of contact force data. This is partly in line with our data, where mean contact force and force time integral were comparable between both groups. However, in our cohort, $80 \%$ of ablation points reached a target contact force $\geq 10 \mathrm{~g}$, irrespective of randomization. A possible explanation might be different anatomical reachability of the pulmonary veins compared to the LA anterior wall.

4.2. Visibility of the Anterior Line on $c M R I$. Patients underwent cMRI shortly before and three to six months after ablation with the aim of visualizing the LA anterior line [15] and to associate it with the applied contact force. In $81 \%$ of cMRIs after ablation, the LA anterior line was visible in at least one segment. However, a visible line in all three segments could only be detected in $52 \%$ of patients. This 
contrasts a bidirectional electrical block that was achievable in $92 \%$ of patients. Similarly in a prior study, ablation lines could only be recognized in $54 \%$ of patients using cMRI with late gadolinium enhancement [16]. In our cohort, patients without visible lines in all three segments did not show a lower contact force compared to patients with a visible line. This is in contrast to Andreu et al., where gaps in the ablation lines around the pulmonary veins correlated with reduced contact force (6.7 versus 12.2g) in 36 patients [17]. As in our cohort, $80 \%$ of ablation points had a contact force $\geq 10 \mathrm{~g}$. Radiofrequency applications with low contact force at the anterior wall were few and a significant difference might have been missed. However, other studies were not able to substantiate a clear correlation between ablation gaps and scar tissue on cMRI either [18]. Such heterogeneity implies that cMRI image acquisition and postprocessing are variable across studies, and further efforts are required to standardize and harmonize cMRI protocols for future studies and to improve lesion imaging.

4.3. Success at 12-Month Follow-Up. After systematic 12-month follow-up including repetitive 7-day Holter-monitoring and detailed questioning, we did not find a significant difference in AF recurrence and single procedure success between both groups. This is in line with existing work, where the use of contact force did not improve the rate of sinus rhythm after midterm follow-up $[6,12,13,19]$. Also, a 1-year success rate of about $50 \%$ in patients undergoing ablation for persistent $\mathrm{AF}$ appears to be comparable to others [10]. New technologies like ablation index [20] and use of higher contact fore or the use of high power ablation might improve ablation outcome. However, prospective randomized trials on this subject are still under way.

\section{Limitations}

Our results clearly show no relevant differences between our study groups randomized to LA anterior line ablation with or without display of contact force information. It appears unlikely that a larger cohort would have relevantly changed our results. However, we submit that we did not observe the anticipated difference in procedure time that was the basis for our power calculation. It is thus possible that we have missed significant differences due to a lack of statistical power. Complete cMRI data before and after the ablation procedure was only available in 50 patients with no difference between both groups (visible group: 24 versus blinded group: $26, \mathrm{p}=0.45$ ). This might have further reduced our statistical power for cMRI specific analyses. In most of the procedures, we did not use a deflectable sheath for the ablation catheter; perhaps this would have improved the efficacy of the linear ablation. However, $97 \%$ of bidirectional block is higher than previously published.

\section{Conclusion}

In this prospective randomized clinical trial, information on contact force during the ablation of a LA anterior line did not reduce radiofrequency time to achieve bidirectional line block. In addition, other procedural data as well as the ablation success rate after 12 months of follow-up did not reveal significant differences between both groups. By cMRI, LA anterior line visibility did not significantly correlate with the applied contact force. In conclusion, information on contact force does not seem to improve procedural and overall ablation success with respect to LA anterior line ablation. In addition, creating anterior line as the only additional lesion in patients with persistent AF in the attempt to improve the success rate of the procedure is still disputable and, therefore, it just remains a partial technique, due to the complexity of the pathophysiology of persistent AF.

\section{Data Availability}

The data used to support the findings of this study are available from the corresponding author upon request.

\section{Ethical Approval}

The ethics committee at the Ludwig-Maximilians University, Munich, Germany, approved the study.

\section{Disclosure}

The funders did not play any role in study design, data collection and analysis, decision to publish, or preparation of the manuscript.

\section{Conflicts of Interest}

The authors declare that they have no conflicts of interest.

\section{Acknowledgments}

This work was supported by an investigator initiated grant by Biosense Webster (Grant no. IIS 163).

\section{References}

[1] H. Calkins, K. H. Kuck, R. Cappato et al., "2012 HRS/EHRA/ ECAS expert consensus statement on catheter and surgical ablation of atrial fibrillation: recommendations for patient selection, procedural techniques, patient management and follow-up, definitions, endpoints, and research trial design," Journal of Interventional Cardiac Electrophysiology, vol. 33, no. 2, pp. 171$257,2012$.

[2] T.-H. Kim, J. Park, J.-K. Park et al., "Linear ablation in addition to circumferential pulmonary vein isolation (Dallas lesion set) does not improve clinical outcome in patients with paroxysmal atrial fibrillation: A prospective randomized study," Europace, vol. 17, no. 3, pp. 388-395, 2015.

[3] S. Tzeis, A. Luik, C. Jilek et al., "The modified anterior line: an alternative linear lesion in perimitral flutter," Journal of Cardiovascular Electrophysiology, vol. 21, no. 6, pp. 665-670, 2010.

[4] K. Yokoyama, H. Nakagawa, D. C. Shah et al., "Novel contact force sensor incorporated in irrigated radiofrequency ablation catheter predicts lesion size and incidence of steam pop and thrombus," Circulation: Arrhythmia and Electrophysiology, vol. 1, no. 5, pp. 354-362, 2008. 
[5] A. Thiagalingam, A. D’Avila, and L. Foley, "Importance of catheter contact force during irrigated radiofrequency ablation: evaluation in a porcine ex vivo model using a force-sensing catheter," Journal of Cardiovascular Electrophysiology, vol. 21, no. 7, pp. 806-811, 2010.

[6] W. Ullah, A. McLean, M. H. Tayebjee et al., "Randomized trial comparing pulmonary vein isolation using the SmartTouch catheter with or without real-time contact force data," Heart Rhythm, vol. 13, no. 9, pp. 1761-1767, 2016.

[7] A. Natale, V. Y. Reddy, and G. Monir, “ Paroxysmal AF catheter ablation with a contact force sensing catheter: results of the prospective, multicenter SMART-AF trial," Journal of the American College of Cardiology, vol. 64, pp. 647-656, 2014.

[8] S. Fichtner, K. Sparn, T. Reents et al., "Recurrence of paroxysmal atrial fibrillation after pulmonary vein isolation: Is repeat pulmonary vein isolation enough? A prospective, randomized trial," Europace, vol. 17, no. 9, pp. 1371-1375, 2015.

[9] J. Kautzner, P. Neuzil, H. Lambert et al., "EFFICAS II: Optimization of catheter contact force improves outcome of pulmonary vein isolation for paroxysmal atrial fibrillation," Europace, vol. 17, no. 8, pp. 1229-1235, 2015.

[10] A. Verma, C.-Y. Jiang, T. R. Betts et al., "Approaches to catheter ablation for persistent atrial fibrillation," The New England Journal of Medicine, vol. 372, no. 19, pp. 1812-1822, 2015.

[11] M. Ulbrich, A. Hahnefeld, R. Wakili, S. Clau, N. KS. Oversohl, and S. Clauß, "Einfluss der echtzeitmessung von contact force auf die linksatriale prozedur bei zirkumferentieller pulmonalvenenisolation. Clin Res Cardiol," Einfluss der echtzeitmessung von contact force auf die linksatriale prozedur bei zirkumferentieller pulmonalvenenisolation. Clin Res Cardiol, p. 100, 2011.

[12] V. Y. Reddy, S. R. Dukkipati, and P. Neuzil, "Randomized, controlled trial of the safety and effectiveness of a contact force-sensing irrigated catheter for ablation of paroxysmal atrial fibrillation: results of the TactiCath Contact Force Ablation Catheter Study for Atrial Fibrillation (TOCCASTAR) study," Circulation, vol. 132, no. 10, pp. 907-915, 2015.

[13] P. Neuzil, V. Y. Reddy, J. Kautzner et al., "Electrical reconnection after pulmonary vein isolation is contingent on contact force during initial treatment: results from the EFFICAS i study," Circulation: Arrhythmia and Electrophysiology, vol. 6, no. 2, pp. 327-333, 2013.

[14] K.-H. Kuck, V. Y. Reddy, B. Schmidt et al., "A novel radiofrequency ablation catheter using contact force sensing: Toccata study," Heart Rhythm, vol. 9, no. 1, pp. 18-23, 2012.

[15] R. Ranjan, E. G. Kholmovski, J. Blauer et al., "Identification and acute targeting of gaps in atrial ablation lesion sets using a real-time magnetic resonance imaging system," Circulation: Arrhythmia and Electrophysiology, vol. 5, no. 6, pp. 1130-1135, 2012.

[16] R. J. Hunter, D. A. Jones, R. Boubertakh et al., "Diagnostic accuracy of cardiac magnetic resonance imaging in the detection and characterization of left atrial catheter ablation lesions: A multicenter experience," Journal of Cardiovascular Electrophysiology, vol. 24, no. 4, pp. 396-403, 2013.

[17] D. Andreu, F. Gomez-Pulido, M. Calvo et al., "Contact force threshold for permanent lesion formation in atrial fibrillation ablation: A cardiac magnetic resonance-based study to detect ablation gaps," Heart Rhythm, vol. 13, no. 1, pp. 37-45, 2016.

[18] J. L. Harrison, C. Sohns, N. W. Linton et al., "Response to Letter From Bisbal et al Regarding, "Repeat Left Atrial Catheter Ablation: Cardiac Magnetic Resonance Prediction of Endocardial Voltage and Gaps in Ablation Lesion Sets"," Circulation:
Arrhythmia and Electrophysiology, vol. 8, no. 3, pp. 754-755, 2015.

[19] S. Fichtner, T. Reents, S. Ammar et al., "Pulmonary vein isolation using new technologies to improve ablation lesion formation: Initial results comparing enhanced catheter tip irrigation (Surround Flow ${ }^{\circledR}$ ) with contact force measurement (Smarttouch $\left.{ }^{\circledR}\right)$," Indian Pacing and Electrophysiology Journal, vol. 15, no. 3, pp. 152-157, 2015.

[20] P. Taghji, M. El Haddad, T. Phlips et al., "Evaluation of a Strategy Aiming to Enclose the Pulmonary Veins With Contiguous and Optimized Radiofrequency Lesions in Paroxysmal Atrial Fibrillation: A Pilot Study," JACC: Clinical Electrophysiology, vol. 4, no. 1, pp. 99-108, 2018. 


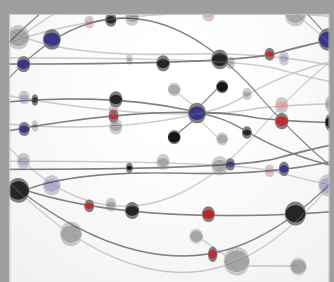

The Scientific World Journal
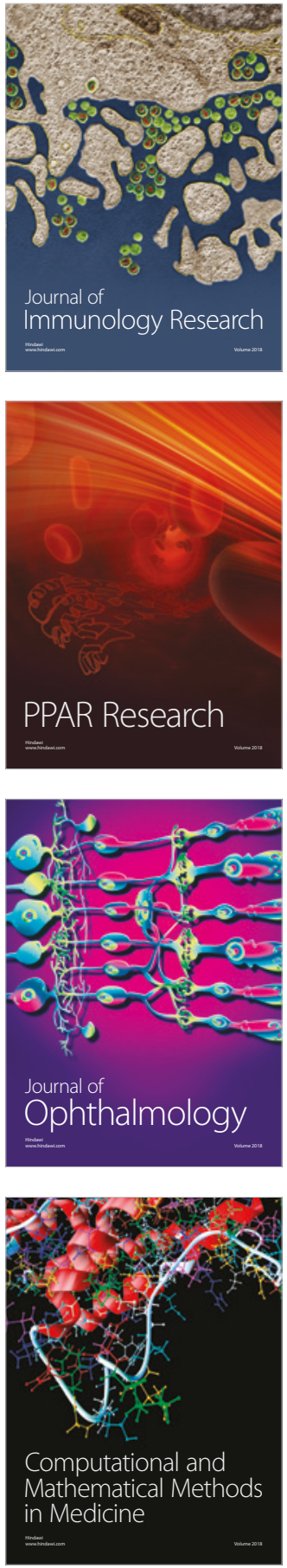

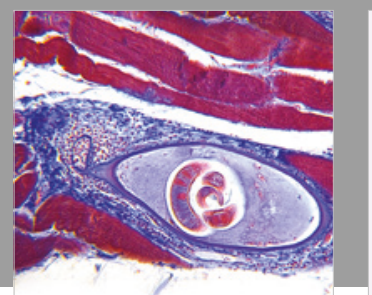

Gastroenterology Research and Practice

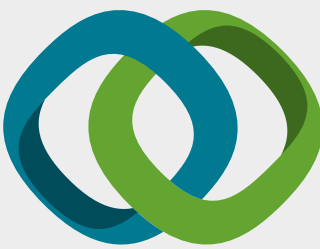

\section{Hindawi}

Submit your manuscripts at

www.hindawi.com
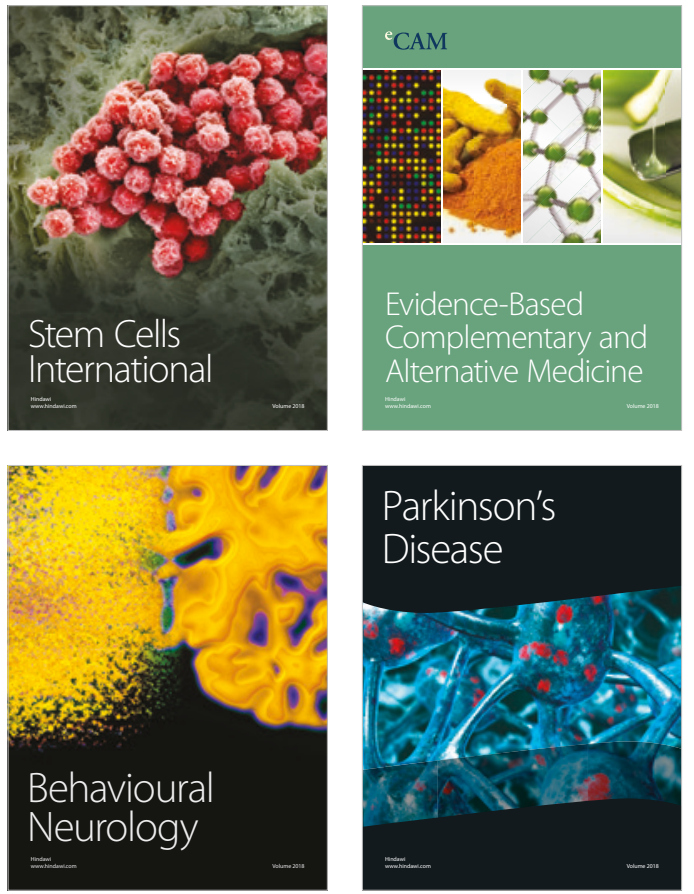

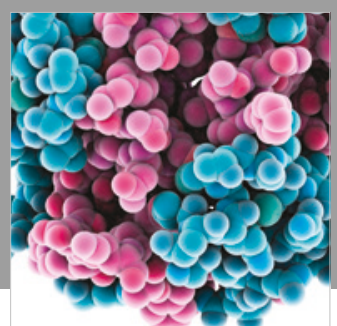

ournal of

Diabetes Research

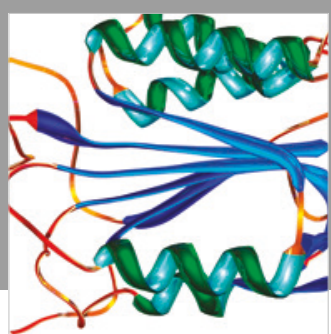

Disease Markers
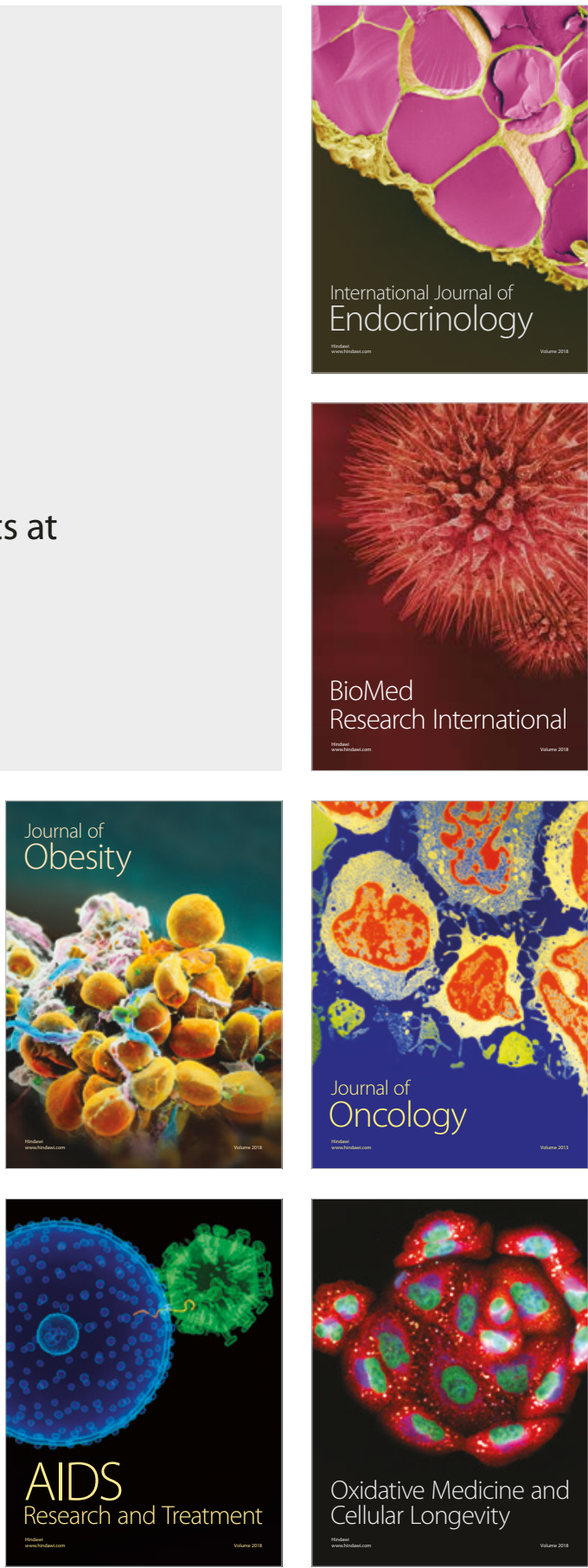